\title{
Article \\ Some Modifications of Pairwise Soft Sets and Some of Their Related Concepts
}

\author{
Samer Al Ghour
}

Citation: Al Ghour, S. Some Modifications of Pairwise Soft Sets and Some of Their Related Concepts. Mathematics 2021, 9, 1781. https:// doi.org/10.3390/math9151781

Academic Editor: Francisco Gallego Lupiaňez

Received: 23 June 2021

Accepted: 17 July 2021

Published: 28 July 2021

Publisher's Note: MDPI stays neutral with regard to jurisdictional claims in published maps and institutional affiliations.

Copyright: (c) 2021 by the authors. Licensee MDPI, Basel, Switzerland. This article is an open access article distributed under the terms and conditions of the Creative Commons Attribution (CC BY) license (https:// creativecommons.org/licenses/by/ $4.0 /)$.
Department of Mathematics and Statistics, Jordan University of Science and Technology, Irbid 22110, Jordan; algore@just.edu.jo

\begin{abstract}
In this paper, we first define soft $u$-open sets and soft s-open as two new classes of soft sets on soft bitopological spaces. We show that the class of soft $p$-open sets lies strictly between these classes, and we give several sufficient conditions for the equivalence between soft $p$-open sets and each of the soft $u$-open sets and soft $s$-open sets, respectively. In addition to these, we introduce the soft $u$ - $\omega$-open, soft $p$ - $\omega$-open, and soft $s-\omega$-open sets as three new classes of soft sets in soft bitopological spaces, which contain soft $u$-open sets, soft $p$-open sets, and soft $s$-open sets, respectively. Via soft $u$-open sets, we define two notions of Lindelöfeness in SBTSs. We discuss the relationship between these two notions, and we characterize them via other types of soft sets. We define several types of soft local countability in soft bitopological spaces. We discuss relationships between them, and via some of them, we give two results related to the discrete soft topological space. According to our new concepts, the study deals with the correspondence between soft bitopological spaces and their generated bitopological spaces.
\end{abstract}

Keywords: soft bitopological space; soft $p$-open sets; $u$-open sets; $s$-open sets; soft $\omega$-open sets; soft Lindelöf; soft locally countable; generated soft topology

\section{Introduction and Preliminaries}

This paper follows the notions and terminologies that appeared in [1,2]. In this paper, TS (resp. STS, BTS, SBTS) means topological space (resp. soft topological space, bitopological space, and soft bitopological space). For the purpose of dealing with uncertain objects, Molodtsov defined soft sets [3] in 1999. Let $U$ be a universal set and $E$ be a set of parameters. A soft set over $U$ relative to $E$ is a function $G: E \longrightarrow \mathcal{P}(U)$, where $\mathcal{P}(U)$ denotes the power set of $U$. $S S(U, E)$ denotes the family of all soft sets over $U$ relative to $E$. The family $\{M \in S S(U, E): M(e)$ is countable for all $e \in E\}$ and is denoted by $C S S(U, E)$. $0_{E}$ and $1_{E}$ denotes the null soft set and the absolute soft set, respectively. The notion of STSs was defined in [4] as follows: the triplet $(U, \tau, E)$, where $\tau \subseteq S S(U, E)$, is called a STS if $\tau$ contains $0_{E}$ and $1_{E}, \tau$ is closed under arbitrary soft union, and $\tau$ is closed under finite soft intersection. For a STS $(U, \tau, E)$, the members $\tau$ are called soft open sets. Soft topological concepts and their applications are still a hot area of research [1,2,5-22]. The concept of $\omega$-open sets in TSs is defined in [23] as follows: let $(U, \Im)$ be a TS and $V \subseteq U$, then $V$ is $\omega$-open set in $(U, \Im)$ if for each $x \in V$, there is $W \in \Im$ such that $x \in W$ and $W-V$ is countable, or equivalently, $V$ is $\omega$-open set in $(U, \Im)$ if and only if for each $x \in V$, there is $W \in \Im$ and a countable set $C \subseteq U$ such that $x \in W-C \subseteq V$. Denote the family of all $\omega$-open sets in the TS $(U, \Im)$ by $\Im_{\omega}$. It is known that $\Im_{\omega}$ forms a topology on $U$ finer than $\Im$. Research via this concept is continued by several researchers (see [24-29]). Three types of $\omega$-open sets were defined and studied in bitopological spaces in [30]. Recently, soft $\omega$-open sets were defined and investigated in STSs in [2], and research via them was continued in $[5,31]$. As a generalization of BTSs and as an extension of STSs, SBTSs were defined and investigated in [32]. The area of research in SBTSs is still hot (see [33-35]). The concept of soft pairwise open (soft $p$-open) sets in SBTSs was defined and studied 
in [36]. Via soft $p$-open sets, several soft bitopological concepts were introduced by various researchers.

In this paper, we first define soft $u$-open sets and soft $s$-open as two new classes of soft sets on soft bitopological spaces, and we show that the class of soft $p$-open sets lies strictly between these classes. Then, we give several sufficient conditions for the equivalence between soft $p$-open sets and each of the soft $u$-open sets and soft $s$-open sets, respectively. In addition to these, we introduce the soft $u-\omega$-open, soft $p$ - $\omega$-open, and soft $s-\omega$-open sets as three new classes of soft sets in soft bitopological spaces, which contain soft $u$-open sets, soft $p$-open sets, and soft $s$-open sets, respectively, and also represent generalizations of the corresponding concepts in BTSs. Via soft $u$-open sets, we define two notions of Lindelöfeness in SBTSs. We discuss the relationship between these two notions, and we characterize these notions via other types of soft sets. We define several types of soft local countability in soft bitopological spaces. We discuss relationships between them, and via some of them, we give two results related to the discrete soft topological space. According to our new concepts, the study deals with the correspondence between soft bitopological spaces and their generated bitopological spaces.

This paper not only can form the theoretical basis for further applications of soft bitopology, such as soft $u$-mappings, soft $s$-mappings, $u$ - $\omega$-mappings, soft $p$ - $\omega$-mappings, soft $s$ - $\omega$-mappings, soft $u$-compactness, soft $s$-compactness, and so on, but also lead to the development of information systems.

For any SBTS $(X, \Im, \aleph), U \in \Im \cup \aleph$ is equivalent to $(U \in \Im$ or $U \in \aleph)$.

The following definitions and results are used in the sequel:

Definition 1. Let $(X, \Im, \aleph)$ be a BTS and let $U \subseteq X$. Then, $U$ is said to be the following:

(a) u-open in $(X, \Im, \aleph)$ if $U \in \Im \cup \aleph[37]$.

(b) Pairwise open (p-open) in $(X, \Im, \aleph)$ if $U=A \cup B$, for some $A \in \Im$ and $B \in \aleph$ [38].

(c) s-open in $(X, \Im, \aleph)$ if it is open in the least upper bound topology on $X$ of $\Im$ and $\aleph$ [38]. Equivalently, $U$ is s-open in $(X, \Im, \aleph)$ if and only if for every $x \in U$, there exist $A \in \Im$ and $B \in \aleph$ such that $x \in A \cap B \subseteq U$.

The family of all $p$-open sets (resp. $u$-open sets, s-open) in $(X, \Im, \aleph)$ is denoted by $p(\Im, \aleph)($ resp. $u(\Im, \aleph),\langle\Im, \aleph\rangle)$.

Theorem 1 ([30]). For any BTS $(X, \Im, \aleph),\{\varnothing, X\} \subseteq u(\Im, \aleph) \subseteq p(\Im, \aleph) \subseteq\langle\Im, \aleph\rangle$.

Definition 2 ([30]). Let $(X, \Im, \aleph)$ be a BTS and let $U \subseteq X$. Then, $U$ is said to be the following:

(a) $u$-w-open in $(X, \Im, \aleph)$ if $U \in \Im_{\omega} \cup \aleph_{\omega}$.

(b) $\quad p$-w-open in $(X, \Im, \aleph)$ if $U=A \cup B$, for some $A \in \Im_{\omega}$ and $B \in \aleph \omega$.

(c) $s$ - $\omega$-open in $(X, \Im, \aleph)$ if $U \in\left\langle\Im_{\omega}, \aleph \omega\right\rangle$.

The family of all $p-\omega$-open sets (resp. $u$ - $\omega$-open sets) in $(X, \Im, \aleph)$ is denoted by $p \omega(\Im, \aleph)($ resp. $u \omega(\Im, \aleph))$.

Theorem 2 ([30]). For any BTS $(X, \Im, \aleph)$ we have the following:

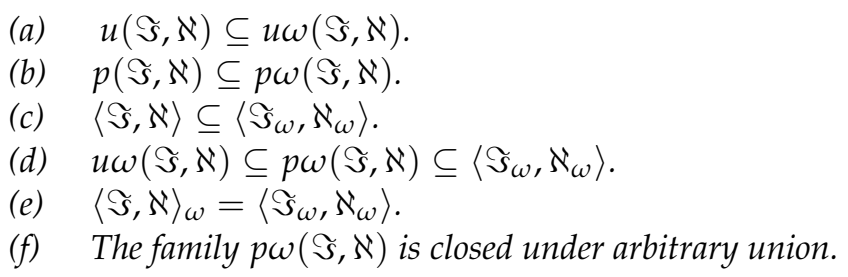

Definition 3 ([39]). Let $M, N \in S S(X, A)$.

(a) $M$ is a soft subset of $N$, denoted by $M \subseteq \widetilde{\widetilde{C}} N$, if $M(a) \subseteq N(a)$ for each $a \in A$.

(b) $M$ and $N$ are said to be soft equal, denoted by $F=G$, if $M \widetilde{\subseteq} N$ and $N \widetilde{\subseteq} M$. 
(c) Soft union of $M$ and $N$ is denoted by $M \widetilde{\cup} N$ and defined to be the soft set $M \widetilde{\cup} N \in S S(X, A)$ where $(M \widetilde{\cup} N)(a)=M(a) \cup N(a)$ for each $a \in A$.

(d) The soft intersection of $M$ and $N$ is denoted by $M \widetilde{\cap} N$ and defined to be the soft set $M \widetilde{\cap} N \in$ $S S(X, A)$ where $(M \widetilde{\cap} N)(a)=M(a) \cap N(a)$ for each $a \in A$.

(e) The difference of $M$ and $N$ is denoted by $M-N$ and defined to be the soft set $M-N \in$ $S S(X, A)$, where $(M-N)(a)=M(a)-N(a)$ for each $a \in A$.

Definition 4 ([40]). Let $\Delta$ be an arbitrary indexed set and $\left\{G_{\alpha}: \alpha \in \Delta\right\} \subseteq S S(X, A)$.

(a) The soft union of these soft sets is the soft set denoted by $\underset{\alpha \in \Delta}{\widetilde{V}} G_{\alpha}$ and defined by $\left(\underset{\alpha \in \Delta}{\mathbb{\cup}} G_{\alpha}\right)(a)=$ $\cup_{\alpha \in \Delta} G_{\alpha}(a)$ for each $a \in A$.

(b) The soft intersection of these soft sets is the soft set denoted by $\widetilde{\cap} G_{\alpha}$ and defined by $\left(\widetilde{\cap} G_{\alpha}\right)(a)=\cap_{\alpha \in \Delta} G_{\alpha}(a)$ for each $a \in A$.

Definition 5 ([41]). Let $G \in S S(X, A)$. $G$ is said to be a soft point over $X$ relative to $A$ if there exist $e \in A$ and $x \in X$ such that the following holds:

$$
G(a)=\left\{\begin{array}{cl}
\{x\} & \text { if } a=e \\
\varnothing & \text { if } a \neq e
\end{array} .\right.
$$

We denote $G$ by $e_{x}$. The family of all soft points over $X$ relative to $A$ is denoted by $S P(X, A)$.

Definition 6 ([41]). Let $G \in S S(X, A)$ and $a_{x} \in S P(X, A)$. Then, $a_{x}$ is said to belong to $F$ (notation: $\left.a_{x} \widetilde{\in} G\right)$ if $a_{x} \widetilde{\subseteq} G$, or, equivalently, $a_{x} \widetilde{\in} G$ if and only if $x \in G(a)$.

Theorem $3([4])$. Let $(X, \tau, A)$ be a STS. Then, the collection $\{F(a): F \in \tau\}$ defines a topology on $X$ for every $a \in A$. This topology is denoted by $\tau_{a}$.

\section{Soft $u$-Open Sets and Soft $s$-Open Sets in SBTSs}

Definition 7 ([36]). Let $(X, \tau, \sigma, A)$ be a SBTS and let $F \in S S(X, A)$. Then, $F$ is said to be soft pairwise open (soft p-open) in $(X, \tau, \sigma, A)$ if for every $a_{x} \widetilde{\in} F$ there is $G \in \tau$ such that $a_{x} \widetilde{\in} G \widetilde{\widetilde{C}} F$ or $H \in \sigma$ such that $a_{x} \widetilde{\in} H \widetilde{\subseteq} F$. Equivalently, $F$ is soft $p$-open if and only if $F=G \widetilde{U} H$, for some $G \in \tau$ and $H \in \sigma$.

The family of all soft $p$-open sets in $(X, \tau, \sigma, A)$ is denoted by $p(\tau, \sigma)$.

It is proved in [36] that $p(\tau, \sigma)$ is closed under arbitrary soft union but not closed under finite soft intersection, in general.

Definition 8. Let $(X, \tau, \sigma, A)$ be a SBTS and let $F \in S S(X, A)$. Then $F$ is said to be the following:

(a) soft u-open in $(X, \tau, \sigma, A)$ if $F \in \tau \cup \sigma$.

(b) soft s-open in $(X, \tau, \sigma, A)$ if it is soft open in the least upper bound soft topology on $X$ relative to $A$, of $\tau$ and $\sigma$. Equivalently: $F$ is soft s-open in $(X, \tau, \sigma, A)$ if and only if for every $a_{x} \widetilde{\in} F$, there exist $M \in \tau$ and $N \in \sigma$ such that $a_{x} \widetilde{\in} M \widetilde{\cap} N \widetilde{\widetilde{C}} F$.

The family of all soft $u$-open sets (resp. soft $s$-open sets) in $(X, \tau, \sigma, A)$ is denoted by $u(\tau, \sigma)(\operatorname{resp} .\langle\tau, \sigma\rangle)$.

Remark 1. For any $\operatorname{SBTS}(X, \tau, \sigma, A),\left\{0_{A}, 1_{A}\right\} \subseteq u(\tau, \sigma) \subseteq p(\tau, \sigma) \subseteq\langle\tau, \sigma\rangle$.

Proof. Straightforward.

The following example shows that the inclusion of $u(\tau, \sigma) \subseteq p(\tau, \sigma)$ in Remark 1 is not an equality, in general. 
Example 1. Let $X=\mathbb{R}, A=\mathbb{Z}$. Let $F, G \in S S(X, A)$ defined by $F(a)=(-\infty, 0)$ and $G(a)=(0, \infty)$ for all $a \in A$. Let $\tau=\left\{0_{A}, 1_{A}, F\right\}$ and $\sigma=\left\{0_{A}, 1_{A}, G\right\}$. Consider the SBTS $(X, \tau, \sigma, A)$. Then $F \widetilde{\cup} G \in p(\tau, \sigma)-u(\tau, \sigma)$.

Remark 2. Example 1 shows also that $u(\tau, \sigma)$ is not closed under soft union.

Theorem 4. For any SBTS $(X, \tau, \sigma, A)$, the following are equivalent:

(a) $u(\tau, \sigma)=p(\tau, \sigma)$.

(b) $u(\tau, \sigma)$ is closed under arbitrary soft union.

Proof. $(\mathrm{a}) \Longrightarrow(\mathrm{b})$ : Since $p(\tau, \sigma)$ is closed under arbitrary soft union, then by (a), we obtain the result.

(b) $\Longrightarrow($ a): By Remark 1 we have $u(\tau, \sigma) \subseteq p(\tau, \sigma)$. To see that $p(\tau, \sigma) \subseteq u(\tau, \sigma)$, let $F \in p(\tau, \sigma)$. Then, $F=G \widetilde{\cup} H$, for some $G \in \tau$ and $H \in \sigma$. Since $G \in \tau \subseteq u(\tau, \sigma)$ and $H \in \sigma \subseteq u(\tau, \sigma)$, then by (b), we have $F \in u(\tau, \sigma)$.

The following example shows that the inclusion $p(\tau, \sigma) \subseteq\langle\tau, \sigma\rangle$ in Remark 1 is not an equality, in general.

Example 2. Let $X=\mathbb{R}, A=\mathbb{N}$,

$$
\tau=\left\{0_{A}, 1_{A}\right\} \cup\{F \in S S(X, A): \text { for every } a \in A, F(a)=(-\infty, b) \text { for some } b \in \mathbb{R}\},
$$

and

$$
\sigma=\left\{0_{A}, 1_{A}\right\} \cup\{F \in S S(X, A): \text { for every } a \in A, F(a)=(b, \infty) \text { for some } b \in \mathbb{R}\} .
$$

Consider the SBTS $(X, \tau, \sigma, A)$. Let $F, M, N \in S S(X, A)$, where $F(a)=(0,5), M(a)=$ $(-\infty, 5)$ and $N(a)=(0, \infty)$ for all $a \in A$. Then, $F=M \widetilde{\cap} N \in\langle\tau, \sigma\rangle-p(\tau, \sigma)$.

Remark 3. Example 2 shows also that $p(\tau, \sigma)$ is not closed under finite soft intersection.

Theorem 5. For any SBTS $(X, \tau, \sigma, A)$, the following are equivalent:

(a) $p(\tau, \sigma)=\langle\tau, \sigma\rangle$.

(b) For every $F, G \in p(\tau, \sigma), F \widetilde{\cap} G \in p(\tau, \sigma)$.

(c) $(X, p(\tau, \sigma), A)$ is a STS.

Proof. (a) $\Longrightarrow$ (b): If $p(\tau, \sigma)=\langle\tau, \sigma\rangle$, then $p(\tau, \sigma)$ is a soft topology on $X$ relative to $A$, and then it is closed with respect to finite soft intersections.

$(b) \Longrightarrow(c)$ : Follows from (b), Remark 1 , and the fact that $p(\tau, \sigma)$ is closed under arbitrary soft union.

(c) $\Longrightarrow$ (a): By Remark 1, we have $p(\tau, \sigma) \subseteq\langle\tau, \sigma\rangle$. To see that $\langle\tau, \sigma\rangle \subseteq p(\tau, \sigma)$, it is sufficient to show that $M \widetilde{\cap} N \in p(\tau, \sigma)$ whenever $M \in \tau$ and $N \in \sigma$. Let $M \in \tau$ and $N \in \sigma$, then by Remark $1, M, N \in p(\tau, \sigma)$. So by (c), $M \widetilde{\cap} N \in p(\tau, \sigma)$.

Theorem 6. Let $(X, \tau, \sigma, A)$ be a SBTS and let $F \in S S(X, A)$. Then, we have the following:

(a) If $F \in u(\tau, \sigma)$, then $F(a) \in u\left(\tau_{a}, \sigma_{a}\right)$ for all $a \in A$.

(b) If $F \in p(\tau, \sigma)$, then $F(a) \in p\left(\tau_{a}, \sigma_{a}\right)$ for all $a \in A$.

(c) If $F \in\langle\tau, \sigma\rangle$, then $F(a) \in\left\langle\tau_{a}, \sigma_{a}\right\rangle$ for all $a \in A$.

(d) $\langle\tau, \sigma\rangle_{a}=\left\langle\tau_{a}, \sigma_{a}\right\rangle$ for all $a \in A$.

Proof. (a) Suppose that $F \in u(\tau, \sigma)$, and let $a \in A$. Since $F \in u(\tau, \sigma)=\tau \cup \sigma$ and so $F \in \tau$ or $F \in \sigma$. Thus, we have $F(a) \in \tau_{a}$ or $F(a) \in \sigma_{a}$, and hence $F(a) \in \tau_{a} \cup \sigma_{a}=u\left(\tau_{a}, \sigma_{a}\right)$. 
(b) Suppose that $F \in p(\tau, \sigma)$ and let $a \in A$. Since $F \in p(\tau, \sigma)$, then $F=M \widetilde{\cup} N$ where $M \in \tau$ and $N \in \sigma$. So, $F(a)=M(a) \cup N(a)$. Since $M \in \tau$ and $N \in \sigma$, then $M(a) \in \tau_{a}$ and $N(a) \in \sigma_{a}$. This shows that $F(a) \in p\left(\tau_{a}, \sigma_{a}\right)$.

(c) Suppose that $F \in\langle\tau, \sigma\rangle$ and let $a \in A$. To show that $F(a) \in\left\langle\tau_{a}, \sigma_{a}\right\rangle$ let $x \in F(a)$, then $a_{x} \widetilde{\in} F$. So, there exist $M \in \tau$ and $N \in \sigma$ such that $a_{x} \widetilde{\in} M \widetilde{\cap} N \widetilde{\widetilde{F}} F$. This implies that $x \in M(a) \cap N(a) \subseteq F(a)$ with $M(a) \in \tau_{a}$ and $N(a) \in \sigma_{a}$. Hence, $F(a) \in\left\langle\tau_{a}, \sigma_{a}\right\rangle$.

(d) Let $a \in A$. To see that $\langle\tau, \sigma\rangle_{a} \subseteq\left\langle\tau_{a}, \sigma_{a}\right\rangle$, let $W \in\langle\tau, \sigma\rangle_{a}$. Then, there exists $G \in\langle\tau, \sigma\rangle$ such that $G(a)=W$. By (c), $G(a) \in\left\langle\tau_{a}, \sigma_{a}\right\rangle$ and so $W \in\left\langle\tau_{a}, \sigma_{a}\right\rangle$. To see that $\left\langle\tau_{a}, \sigma_{a}\right\rangle \subseteq\langle\tau, \sigma\rangle_{a}$, let $W \in\left\langle\tau_{a}, \sigma_{a}\right\rangle-\{\varnothing\}$ and let $x \in W$. Then, there are $U \in \tau_{a}$ and $V \in \sigma_{a}$ such that $x \in U \cap V \subseteq W$. Choose $M \in \tau$ and $N \in \sigma$ such that $U=M(a)$ and $V=N(a)$. Thus, we have $M \widetilde{\cap} N \in\langle\tau, \sigma\rangle$ and so $(M \widetilde{\cap} N)(a)=M(a) \cap N(a)=U \cap V \in\langle\tau, \sigma\rangle_{a}$. This shows that $W \in\langle\tau, \sigma\rangle_{a}$.

The following example shows that each of the three implications in Theorem 6 is not reversible, in general.

Example 3. Let $X=\mathbb{R}, A=\{a, b\}$. Let $F, G \in S S(X, A)$ defined by the following:

$$
\begin{aligned}
& F(a)=\{1\}, F(b)=\varnothing \\
& G(a)=\{1\}, G(b)=\mathbb{R} .
\end{aligned}
$$

Consider the SBTS $(X, \tau, \sigma, A)$, where $\tau=\sigma=\left\{0_{A}, 1_{A}, F\right\}$. Then $\tau_{a}=\sigma_{a}=\{\varnothing, X,\{1\}\}$ and $\tau_{b}=\sigma_{b}=\{\varnothing, X\}$. Thus we have $G(a)=\{1\} \in \tau_{a}=\sigma_{a}=u\left(\tau_{a}, \sigma_{a}\right)=p\left(\tau_{a}, \sigma_{a}\right)=\left\langle\tau_{a}, \sigma_{a}\right\rangle$ and $G(b)=\mathbb{R} \in \tau_{b}=\sigma_{b}=u\left(\tau_{b}, \sigma_{b}\right)=p\left(\tau_{b}, \sigma_{b}\right)=\left\langle\tau_{b}, \sigma_{b}\right\rangle$, while $G \notin \tau=\sigma=u(\tau, \sigma)=$ $p(\tau, \sigma)=\langle\tau, \sigma\rangle$.

\section{Soft $u-\omega$-Openess, $\boldsymbol{p}$ - $\boldsymbol{\omega}$-Openess and $\boldsymbol{s}-\boldsymbol{\omega}$-Openess in SBTSs}

Definition 9. Let $(X, \tau, \sigma, A)$ be a SBTS and let $F \in S S(X, A)$. Then $F$ is said to be the following:

(a) soft $u$ - $\omega$-open in $(X, \tau, \sigma, A)$ if $F \in \tau_{\omega} \cup \sigma_{\omega}$. Equivalently, $F$ is soft $u$ - $\omega$-open in $(X, \tau, \sigma, A)$ if and only if $F \in u\left(\tau_{\omega}, \sigma_{\omega}\right)$.

(b) soft pairwise $\omega$-open (soft $p$ - $\omega$-open) in $(X, \tau, \sigma, A)$ if for every $a_{x} \tilde{\in} F$ there is $G \in \tau_{\omega}$ such that $a_{x} \widetilde{\in} G \widetilde{\subseteq} F$ or $H \in \sigma_{\omega}$ such that $a_{x} \widetilde{\in} H \widetilde{\subseteq} F$. Equivalently, $F$ is soft $p$ - $\omega$-open if and only if $F \in p\left(\tau_{\omega}, \sigma_{\omega}\right)$

(c) soft s-w-open in $(X, \tau, \sigma, A)$ if it is soft open in the least upper bound soft topology on $X$ relative to $A$, of $\tau_{\omega}$ and $\sigma_{\omega}$. Equivalently, $F$ is soft s- $\omega$-open in $(X, \tau, \sigma, A)$ if and only if $F \in$ $\left\langle\tau_{\omega}, \sigma_{\omega}\right\rangle$.

The family of all soft $u$ - $\omega$-open (resp. $p$ - $\omega$-open) sets in $(X, \tau, \sigma, A)$ is denoted by $u \omega(\tau, \sigma)(\operatorname{resp} . p \omega(\tau, \sigma))$.

Theorem 7. For any SBTS $(X, \tau, \sigma, A)$, we have the following:

(a) $u(\tau, \sigma) \subseteq u \omega(\tau, \sigma)$.

(b) $p(\tau, \sigma) \subseteq p \omega(\tau, \sigma)$.

(c) $\langle\tau, \sigma\rangle \subseteq\left\langle\tau_{\omega}, \sigma_{\omega}\right\rangle$.

(d) $\quad u \omega(\tau, \sigma) \subseteq p \omega(\tau, \sigma) \subseteq\left\langle\tau_{\omega}, \sigma_{\omega}\right\rangle$.

(e) $\langle\tau, \sigma\rangle_{\omega}=\left\langle\tau_{\omega}, \sigma_{\omega}\right\rangle$.

(f) The family $p \omega(\tau, \sigma)$ is closed under arbitrary soft union.

Proof. (a), (b) and (c) are obvious.

(d) By Remark 1, we have $u \omega(\tau, \sigma)=u\left(\tau_{\omega}, \sigma_{\omega}\right) \subseteq p\left(\tau_{\omega}, \sigma_{\omega}\right)=p \omega(\tau, \sigma) \subseteq\left\langle\tau_{\omega}, \sigma_{\omega}\right\rangle$.

(e) Let $F \in\langle\tau, \sigma\rangle_{\omega}$ and let $a_{x} \widetilde{\in} F$, then there exist $G \in\langle\tau, \sigma\rangle$ and $H \in \operatorname{CSS}(X, A)$ such that $a_{x} \widetilde{\in} G-H \widetilde{\simeq} F$. Since $a_{x} \widetilde{\in} G \in\langle\tau, \sigma\rangle$, then there exist $M \in \tau$ and $N \in \sigma$ such that $a_{x} \in$ $M \widetilde{\cap} N \widetilde{\subseteq} G$. So, we have $M-H \in \tau_{\omega}, N-H \in \sigma_{\omega}$, and $a_{x} \widetilde{\in}(M-H) \widetilde{\cap}(N-H) \widetilde{\subseteq}(M \widetilde{\cap} N)-$ 
$H \widetilde{\subseteq} G-H \widetilde{\subseteq} F$. Hence, $F \in\left\langle\tau_{\omega}, \sigma_{\omega}\right\rangle$. Conversely, let $F \in\left\langle\tau_{\omega}, \sigma_{\omega}\right\rangle$ and let $a_{x} \widetilde{\in} F$. Then, there exist $W \in \tau_{\omega}$ and $L \in \sigma_{\omega}$ such that $a_{x} \widetilde{\in} W \widetilde{\cap} L \widetilde{\widetilde{C}} F$. Since $a_{x} \widetilde{\in} W \widetilde{\cap} L$, then there exist $M \in \tau$, $N \in \sigma$ and $H, K \in \operatorname{CSS}(X, A)$ such that $a_{x} \widetilde{\in} M-H \widetilde{\subseteq} W$ and $a_{x} \widetilde{\in} N-K \widetilde{\subseteq} L$. Note that $M \widetilde{\cap} N \in\langle\tau, \sigma\rangle$ and $H \widetilde{\cap} K \in \operatorname{CSS}(X, A)$. Additionally, $a_{x} \widetilde{\in}(M \widetilde{\cap} N)-(H \widetilde{\cap} K) \subseteq W \widetilde{\cap} L \subseteq F$. Thus, $F \in\langle\tau, \sigma\rangle_{\omega}$.

(f) Since $p \omega(\tau, \sigma)=p\left(\tau_{\omega}, \sigma_{\omega}\right)$ and $p\left(\tau_{\omega}, \sigma_{\omega}\right)$ is closed under arbitrary soft union, we obtain the result.

The inclusions in parts (a), (b) and (c) of Theorem 7 are not equalities, in general.

Example 4. Let $X=A=\mathbb{N}, \tau=\sigma=\left\{0_{A}, 1_{A}\right\}, a=x=1$. Consider the SBTS $(X, \tau, \sigma, A)$. Then $a_{x} \in u \omega(\tau, \sigma)=p \omega(\tau, \sigma)=\left\langle\tau_{\omega}, \sigma_{\omega}\right\rangle=\tau_{\omega}$, but $a_{x} \notin u(\tau, \sigma)=p(\tau, \sigma)=\langle\tau, \sigma\rangle=\tau$.

The inclusions in Theorem 7 (d) are not equalities, in general, as the following two examples show:

Example 5. Let $X=\mathbb{R}, A=\mathbb{Z}$. Let $F, G \in S S(X, A)$ defined by $F(a)=(-\infty, 0)$ and $G(a)=(0, \infty)$ for all $a \in A$. Let $\tau=\left\{0_{A}, 1_{A}, F\right\}$ and $\sigma=\left\{0_{A}, 1_{A}, G\right\}$. Consider the SBTS $(X, \tau, \sigma, A)$. Then $F \widetilde{\cup} G \in p \omega(\tau, \sigma)-u \omega(\tau, \sigma)$.

Example 6. Let $X=\mathbb{R}, A=\mathbb{N}$,

$$
\tau=\left\{0_{A}, 1_{A}\right\} \cup\{F \in S S(X, A): \text { for every } a \in A, F(a)=(-\infty, b) \text { for some } b \in \mathbb{R}\},
$$

and

$$
\sigma=\left\{0_{A}, 1_{A}\right\} \cup\{F \in S S(X, A): \text { for every } a \in A, F(a)=(b, \infty) \text { for some } b \in \mathbb{R}\} .
$$

Consider the SBTS $(X, \tau, \sigma, A)$ and let $F \in S S(X, A)$, where $F(a)=(0,5)$ for all $a \in A$. Then, $F \in\langle\tau, \sigma\rangle \subseteq\langle\tau, \sigma\rangle_{\omega}$, but $F \notin p \omega(\tau, \sigma)$.

Remark 4. In Example 5, it is not difficult to check that $F, G \in u \omega(\tau, \sigma)$ while $F \widetilde{\cup} G \notin u \omega(\tau, \sigma)$. This shows that $u \omega(\tau, \sigma)$ is not closed under arbitrary soft union, in general.

Corollary 1. For any SBTS $(X, \tau, \sigma, A)$, the following are equivalent:

(a) $u \omega(\tau, \sigma)=p \omega(\tau, \sigma)$.

(b) $u \omega(\tau, \sigma)$ is closed under arbitrary soft union.

Proof. Follows directly from Theorem 4.

The next example shows that the soft intersection of two soft $p-\omega$-open sets is not soft $p$ - $\omega$-open in general. Therefore, for a given SBTS $(X, \tau, \sigma, A),(X, p \omega(\tau, \sigma), A)$ is not an STS, in general.

Example 7. Consider the SBTS $(X, \tau, \sigma, A)$ as in Example 6. Let $F, G \in S S(X, A)$, where $F(a)=$ $(-\infty, 5)$ and $G(a)=(0, \infty)$ for all $a \in A$. Then $F, G \in p \omega(\tau, \sigma)$, while $F \widetilde{\cap} G \notin p \omega(\tau, \sigma)$.

Corollary 2. For any $\operatorname{SBTS}(X, \tau, \sigma, A)$, the following are equivalent:

(a) $p \omega(\tau, \sigma)=\left\langle\tau_{\omega}, \sigma_{\omega}\right\rangle$.

(b) For every $F, G \in p \omega(\tau, \sigma), F \widetilde{\cap} G \in p \omega(\tau, \sigma)$.

(c) $(X, p \omega(\tau, \sigma), A)$ is a STS.

Proof. Follows directly from Theorem 5.

Theorem 8. Let $(X, \tau, \sigma, A)$ be a SBTS and $F \in S S(X, A)$. Then, $F \in p \omega(\tau, \sigma)$ if and only if for each $a_{x} \widetilde{\in} F$ there exists $G \in u(\tau, \sigma)$ and $H \in \operatorname{CSS}(X, A)$ such that $a_{x} \widetilde{\in} G-H \widetilde{\subseteq} F$. 
Proof. Necessity: Let $F \in p \omega(\tau, \sigma)$ and let $a_{x} \widetilde{\in} F$. Then $F=G \widetilde{U} M$ for some $G \in \tau_{\omega}$ and $M \in \sigma_{\omega}$. Without loss of generality, we may assume that $a_{x} \widetilde{\in} G$. So, there is $L \in \tau \subseteq u(\tau, \sigma)$ and $H \in \operatorname{CSS}(X, A)$ such that $a_{x} \widetilde{\in} L-H \widetilde{\simeq} G \widetilde{\simeq} F$.

Sufficiency: By assumption, for each $a_{x} \widetilde{\in} F$, there exists $G_{a_{x}} \in u(\tau, \sigma)$ and $H_{a_{x}} \in$ $\operatorname{CSS}(X, A)$ such that $a_{x} \widetilde{\in} G_{a_{x}}-H_{a_{x}} \widetilde{\widetilde{C}} F$. Put $S=\widetilde{\cup}\left\{G_{a_{x}}-H_{a_{x}}: G_{a_{x}} \in \tau\right\}$ and $W=\widetilde{\cup}\left\{G_{a_{x}}-H_{a_{x}}: G_{a_{x}} \in \sigma\right\}$. Then $S \in \tau_{\omega}, W \in \sigma_{\omega}$, and $F=S \widetilde{\cup} W$. Hence, $F \in$ $p \omega(\tau, \sigma)$.

Theorem 9. Let $(X, \tau, \sigma, A)$ be a SBTS and let $F \in S S(X, A)$. Then, the following holds.

(a) If $F \in u \omega(\tau, \sigma)$, then $F(a) \in u \omega\left(\tau_{a}, \sigma_{a}\right)$ for all $a \in A$.

(b) If $F \in p \omega(\tau, \sigma)$, then $F(a) \in p \omega\left(\tau_{a}, \sigma_{a}\right)$ for all $a \in A$.

(c) $\quad\left(\langle\tau, \sigma\rangle_{a}\right)_{\omega}=\left(\langle\tau, \sigma\rangle_{\omega}\right)_{a}$ for all $a \in A$.

(d) If $F \in\langle\tau, \sigma\rangle_{\omega}$, then $F(a) \in\left(\langle\tau, \sigma\rangle_{a}\right)_{\omega}$ for all $a \in A$.

Proof. (a) Suppose that $F \in u \omega(\tau, \sigma)$ and let $a \in A$. Since $F \in u \omega(\tau, \sigma)=u\left(\tau_{\omega}, \sigma_{\omega}\right)$, then by Theorem 6 (a), $F(a) \in u\left(\left(\tau_{\omega}\right)_{a},\left(\sigma_{\omega}\right)_{a}\right)$. Thus, by Theorem 7 of [2], $F(a) \in$ $u\left(\left(\tau_{a}\right)_{\omega},\left(\sigma_{a}\right)_{\omega}\right)=u \omega\left(\tau_{a}, \sigma_{a}\right)$.

(b) Suppose that $F \in p \omega(\tau, \sigma)$ and let $a \in A$. Since $F \in p \omega(\tau, \sigma)=p\left(\tau_{\omega}, \sigma_{\omega}\right)$, then by Theorem $6(\mathrm{~b})$, we have $F(a) \in p\left(\left(\tau_{\omega}\right)_{a^{\prime}}\left(\sigma_{\omega}\right)_{a}\right)$ and by Theorem 7 of [2], it follows that $F(a) \in p\left(\left(\tau_{a}\right)_{\omega^{\prime}}\left(\sigma_{a}\right)_{\omega}\right)=p \omega\left(\tau_{a}, \sigma_{a}\right)$.

(c) By Theorem 7 of [2], we have $\left(\langle\tau, \sigma\rangle_{a}\right)_{\omega}=\left(\langle\tau, \sigma\rangle_{\omega}\right)_{a}$ and by Theorem 7 (f) we have $\langle\tau, \sigma\rangle_{\omega}=\left\langle\tau_{\omega}, \sigma_{\omega}\right\rangle$. Thus, we have $\left(\langle\tau, \sigma\rangle_{a}\right)_{\omega}=\left\langle\tau_{\omega}, \sigma_{\omega}\right\rangle_{a}$. By Theorem $6(\mathrm{~d})$, we have $\left\langle\tau_{\omega}, \sigma_{\omega}\right\rangle_{a}=\left\langle\left(\tau_{\omega}\right)_{a},\left(\sigma_{\omega}\right)_{a}\right\rangle$. Therefore, by Theorem 7 (f) and Theorem 7 of [2], $\left(\langle\tau, \sigma\rangle_{a}\right)_{\omega}=$ $\left(\langle\tau, \sigma\rangle_{\omega}\right)_{a}$.

(d) Suppose that $F \in\langle\tau, \sigma\rangle_{\omega}$ and let $a \in A$. Then by parts (c) and (d) of Theorem 6, $F(a) \in\left(\langle\tau, \sigma\rangle_{\omega}\right)_{a}$. Thus, by $(\mathrm{c})$, we have $F(a) \in\left(\langle\tau, \sigma\rangle_{a}\right)_{\omega}$.

The rest of this section focuses on two new types of soft Lindelöfeness SBTSs.

Definition 10. Let $(X, \tau, \sigma, A)$ be a SBTS and let $\mathcal{U} \subseteq S S(X, A)$. Then $\mathcal{U}$ is called the following:

(a) A soft cover of $(X, \tau, \sigma, A)$ if $\widetilde{\cup}\{U: U \in \mathcal{U}\}=1_{A}$.

(b) A soft u-open cover of $(X, \tau, \sigma, A)$ if $\mathcal{U}$ is a soft cover of $(X, \tau, \sigma, A)$ and $\mathcal{U} \subseteq u(\tau, \sigma)$.

(c) A soft v-open cover of $(X, \tau, \sigma, A)$ if it is a soft $u$-open cover, and there are $F \in \tau$ and $G \in \sigma$ such that $\{F, G\} \subseteq \mathcal{U}-\left\{0_{A}\right\}$.

Definition 11. A SBTS $(X, \tau, \sigma, A)$ is called the following:

(a) Soft $u$-Lindelöf if every soft $u$-open cover of $(X, \tau, \sigma, A)$ has a countable subcover.

(b) Soft $v$-Lindelöf if every soft v-open cover of $(X, \tau, \sigma, A)$ has a countable subcover.

Theorem 10. Every soft $u$-Lindelöf SBTS is soft v-Lindelöf.

Proof. Follows from the definition and Remark 1.

The converse of Theorem 10 is not true in general, as can be seen from the following example.

Example 8. Let $X=\mathbb{R}, A$ is any non-empty countable set of parameters, $\tau=S S(X, A)$ and $\sigma=\left\{0_{A}\right\} \cup\{F \in S S(X, A): X-F(a)$ is countable for all $a \in A\}$. Then

(1) $(X, \tau, \sigma, A)$ is soft $v$-Lindelöf: Let $\mathcal{W}$ be soft u-open cover of $(X, \tau, \sigma, A)$. Let $G \in$ $\mathcal{W} \cap\left(\sigma-\left\{0_{A}\right\}\right)$, then we have $\mathbb{R}-G(a)$ is countable for all $a \in A$. For each $a \in A$ and $x \in$ $(\mathbb{R}-G(a))$, choose $F_{a_{x}} \in \mathcal{W}$ such that $a_{x} \tilde{\in} F_{a_{x}} . \quad$ Let $\mathcal{W}_{1}=\{G\} \cup\left\{F_{a_{x}}: a \in A\right.$ and $\left.x \in(\mathbb{R}-G(a))\right\}$, then $\mathcal{W}_{1}$ is a countable subcover of $\mathcal{W}$.

(2) $(X, \tau, \sigma, A)$ is not soft $u$-Lindelöf: let $\mathcal{W}=S S(X, A)$; then, $\mathcal{W}$ is a soft $u$-open cover of $(X, \tau, \sigma, A)$, which has no countable subcover. 
Lemma 1. Let $(X, \tau, \sigma, A)$ be a SBTS such that $A$ is countable, and let $\mathcal{B}=\{F-H: F \in u(\tau, \sigma)$ and $H \in \operatorname{CSS}(X, A)\}$. Then $\left(X, \tau_{\omega}, \sigma_{\omega}, A\right)$ is soft $u$-Lindelöf if and only if every soft cover $\mathcal{W}$ of $(X, \tau, \sigma, A)$ with $\mathcal{W} \subseteq \mathcal{B}$ has a countable subcover.

Proof. Necessity: Suppose $\left(X, \tau_{\omega}, \sigma_{\omega}, A\right)$ is soft $u$-Lindelöf and let $\mathcal{W}$ be a soft cover of $(X, \tau, \sigma, A)$ with $\mathcal{W} \subseteq \mathcal{B}$. Since $\mathcal{W} \subseteq \mathcal{B} \subseteq \tau_{\omega} \cup \sigma_{\omega}$, then $\mathcal{W}$ is soft $u$-open cover of $\left(X, \tau_{\omega}\right.$, $\left.\sigma_{\omega}, A\right)$, and thus, $\mathcal{W}$ has a countable subcover.

Sufficiency: Let $\mathcal{M}=\left\{F_{\alpha}: \alpha \in \Delta\right\}$ be a soft $u$-open cover of $\left(X, \tau_{\omega}, \sigma_{\omega}, A\right)$ with $F_{\alpha} \neq 0_{A}$ for all $\alpha \in \Delta$. For each $\alpha \in \Delta$, there exists an indexed set $\Omega_{\alpha}$ such that $F_{\alpha}=$ $\widetilde{\cup}\left\{G_{\beta}-H_{\beta}: \beta \in \Omega_{\alpha}\right\}$ where $G_{\beta} \in \tau \cup \sigma$ and $H_{\beta} \in \operatorname{CSS}(X, A), \beta \in \Omega_{\alpha}$. Put $\mathcal{W}=\left\{G_{\beta}-\right.$ $\left.H_{\beta}: \beta \in \cup\left\{\Omega_{\alpha}: \alpha \in \Delta\right\}\right\}$. Then $\mathcal{W}$ is a soft cover of $(X, \tau, \sigma, A)$ with $\mathcal{W} \subseteq \mathcal{B}$, and by assumption it has a countable subcover. This implies that $\mathcal{M}$ has also a countable subcover.

Theorem 11. For any $\operatorname{SBTS}(X, \tau, \sigma, A)$ with $A$ countable, the following are equivalent:

(a) $(X, \tau, \sigma, A)$ is soft $u$-Lindelöf.

(b) $\left(X, \tau_{\omega}, \sigma_{\omega}, A\right)$ is soft $u$-Lindelöf.

(c) Each soft cover of $(X, \tau, \sigma, A)$ consisting of members of $p \omega(\tau, \sigma)$ has a countable subcover.

(d) Each soft cover of $(X, \tau, \sigma, A)$ consisting of members of $p(\tau, \sigma)$ has a countable subcover.

Proof. (a) $\Longrightarrow$ (b): Suppose that $(X, \tau, \sigma, A)$ is soft $u$-Lindelöf. We apply Lemma 1 . Let $\mathcal{M}=\left\{G_{\alpha}-H_{\alpha}: \alpha \in \Delta\right.$, where $G_{\alpha} \in u(\tau, \sigma)$ and $\left.H_{\alpha} \in \operatorname{CSS}(X, A)\right\}$ be a soft cover of $(X, \tau, \sigma, A)$. Since $\underset{\alpha \in \Delta}{\widetilde{U}} G_{\alpha}=1_{A}$, then by (a), there exists a countable set $\Delta^{\prime} \subseteq \Delta$ such that $\left\{G_{\alpha}: \alpha \in \Delta^{\prime}\right\}$ is a soft cover of $1_{A}$. Put $S=\widetilde{\cap}\left\{H_{\alpha}: \alpha \in \Delta^{\prime}\right\}$ and for each $a_{x} \widetilde{\in} S$, choose $\alpha_{a_{x}} \in \Delta$ such that $a_{x} \widetilde{\in}\left(G_{\alpha_{a_{x}}}-H_{\alpha_{a_{x}}}\right)$. Thus, $\left\{G_{\alpha}-H_{\alpha}: \alpha \in \Delta^{\prime}\right\} \cup\left\{G_{\alpha_{a_{x}}}-H_{\alpha_{a_{x}}}: a_{x} \widetilde{\in} S\right\}$ is a countable subcover of $\mathcal{M}$.

(b) $\Rightarrow$ (c) Suppose that $\left(X, \tau_{\omega}, \sigma_{\omega}, A\right)$ is $u$-Lindelöf. Let $\mathcal{M}=\left\{F_{\alpha}: \alpha \in \Delta\right\}$ be a soft cover of $(X, \tau, \sigma, A)$ consisting of members of $p \omega(\tau, \sigma)$. For each $\alpha \in \Delta$, there exist $G_{\alpha} \in \tau_{\omega}$ and $K_{\alpha} \in \sigma_{\omega}$ such that $F_{\alpha}=G_{\alpha} \cup K_{\alpha}$. Put $\mathcal{A}=\left\{G_{\alpha}: \alpha \in \Delta\right\} \cup\left\{K_{\alpha}: \alpha \in \Delta\right\}$. Then $\mathcal{A}$ is a soft $u$-open cover of $\left(X, \tau_{\omega}, \sigma_{\omega}, A\right)$. So by (b), there is a countable set $\Delta^{\prime} \subseteq \Delta$ such that $\left\{G_{\alpha}: \alpha \in \Delta^{\prime}\right\} \cup\left\{K_{\alpha}: \alpha \in \Delta^{\prime}\right\}$ is a subcover of $\mathcal{A}$. Thus, $\left\{F_{\alpha}: \alpha \in \Delta^{\prime}\right\}$ is a countable subcover of $\mathcal{M}$.

(c) $\Rightarrow$ (d) Follows because $p(\tau, \sigma) \subseteq p \omega(\tau, \sigma)$.

(d) $\Rightarrow$ (a) Follows because $u(\tau, \sigma) \subseteq p(\tau, \sigma)$.

Theorem 12. For a SBTS $(X, \tau, \sigma, A)$ with $A$ countable, the following are equivalent:

(a) $(X, \tau, \sigma, A)$ is soft $v$-Lindelöf,

(b) $\left(X, \tau_{\omega}, \sigma_{\omega}, A\right)$ is soft $v$-Lindelöf.

Proof. (a) $\Longrightarrow$ (b) Suppose that $(X, \tau, \sigma, A)$ is soft $v$-Lindelöf. Let $\mathcal{M}=\left\{M_{\alpha}: \alpha \in \Delta\right\}$ be a soft $v$-open cover of $\left(X, \tau_{\omega}, \sigma_{\omega}, A\right)$. Take $\alpha_{1}, \alpha_{2} \in \Delta$ such that $M_{\alpha_{1}} \in \tau_{\omega}-\left\{0_{A}\right\}, M_{\alpha_{2}} \in$ $\sigma_{\omega}-\left\{0_{A}\right\}$. For each $\alpha \in \Delta$, there exists an indexed set $\Omega_{\alpha}$ such that $M_{\alpha}=\underset{\beta \in \Omega_{\alpha}}{\widetilde{\cup}}\left(G_{\beta}-H_{\beta}\right)$ where $\left\{H_{\beta}: \beta \in \Omega_{\alpha}\right\} \subseteq \operatorname{CSS}(X, A)$ and $\left(\left\{G_{\beta}: \beta \in \Omega_{\alpha}\right\} \subseteq \tau\right.$ or $\left.\left\{G_{\beta}: \beta \in \Omega_{\alpha}\right\} \subseteq \sigma\right)$. For every $i=1,2$, choose $\beta_{i} \in \Omega_{\alpha_{i}}$ such that $G_{\beta_{1}} \in \tau, G_{\beta_{2}} \in \sigma$, and $G_{\beta_{i}} \neq 0_{A}$. Therefore, $\left\{G_{\beta}: \beta \in \cup \cup_{\alpha \in \Delta} \Omega_{\alpha}\right\}$ is a soft $v$-open cover of $(X, \tau, \sigma, A)$. Since $(X, \tau, \sigma, A)$ is soft $v$-Lindelöf, there exists a countable set $\Delta^{\prime} \subseteq \Delta$ such that for every $\alpha \in \Delta^{\prime}$, there exists a countable set $\Gamma_{\alpha} \subseteq \Omega_{\alpha}$ such that $\left\{G_{\beta}: \beta \in \underset{\alpha \in \Delta^{\prime}}{\cup} \Gamma_{\alpha}\right\}$ covers $1_{A}$. Put $S=\widetilde{\cap}\left\{H_{\beta}: \beta \in \underset{\alpha \in \Delta^{\prime}}{\cup} \Gamma_{\alpha}\right\}$. Then $S \in \operatorname{CSS}(X, A)$ and $\left(1_{A}-S\right) \widetilde{\subseteq} \widetilde{\cup}\left\{G_{\beta}-H_{\beta}: \beta \in \underset{\alpha \in \Delta^{\prime}}{\cup} \Gamma_{\alpha}\right\}$. For each $a_{x} \widetilde{\in} S$, choose $\alpha_{a_{x}} \in \Delta$ such that $\alpha_{a_{x}} \widetilde{\in} M_{\alpha_{a_{x}}}$. Thus, $\left\{M_{\alpha}: \alpha \in \Delta^{\prime}\right\} \cup\left\{M_{\alpha_{a_{x}}}: a_{x} \widetilde{\in} S\right\}$ is a countable subcover of $\mathcal{M}$. 
(b) $\Rightarrow$ (a) Suppose that $\left(X, \tau_{\omega}, \sigma_{\omega}, A\right)$ is soft $v$-Lindelöf. Let $\mathcal{M}$ be a soft $v$-open cover of $(X, \tau, \sigma, A)$. Then $\mathcal{M}$ is a soft $v$-open cover of $\left(X, \tau_{\omega}, \sigma_{\omega}, A\right)$ and hence it has a countable subcover.

\section{Soft Local Countability}

Recall that a TS $(X, \Im)$ is locally countable if for each $x \in X$ there exists $U \in \Im$ such that $U$ is a countable set and $x \in U$.

Definition 12. A BTS $(X, \Im, \aleph)$ is called

(1) $u$-locally countable if for each $x \in X$ there exists $U \in u(\Im, \aleph)$ such that $U$ is a countable set and $x \in U$.

(2) $\quad$-locally countable if for each $x \in X$ there exists $U \in p(\Im, \aleph)$ such that $U$ is a countable set and $x \in U$.

(3) s-locally countable if the TS $(X,\langle\Im, \aleph\rangle)$ is loally countable.

Theorem 13. A BTS $(X, \Im, \aleph)$ is u-locally countable if and only if it is p-locally countable.

Proof. Necessity: Suppose that $(X, \Im, \aleph)$ is $u$-locally countable, and let $x \in X$. Then, there is $U \in u(\Im, \aleph)$ such that $U$ is a countable set and $x \in U$. Since $u(\Im, \aleph) \subseteq p(\Im, \aleph)$, then $U \in p(\Im, \aleph)$. Hence, $(X, \Im, \aleph)$ is $p$-locally countable.

Sufficiency: Suppose that $(X, \Im, \aleph)$ is $p$-locally countable, and let $x \in X$. Then, there is $U \in p(\Im, \aleph)$ such that $U$ is a countable set and $x \in U$. Since $U \in p(\Im, \aleph)$, there exist $A \in \Im$ and $B \in \aleph$ such that $U=A \cup B$. Therefore, we have $A$ is a countable set with $x \in A \in \Im \subseteq u(\Im, \aleph)$ or we have $B$ is a countable set with $x \in B \in \aleph \subseteq u(\Im, \aleph)$. Hence, $(X, \Im, \aleph)$ is $u$-locally countable.

Theorem 14. Every p-locally countable BTS is s-locally countable.

Proof. Let $(X, \Im, \aleph)$ be $p$-locally countable, and let $x \in X$. Then there is $U \in p(\Im, \aleph)$ such that $U$ is a countable set and $x \in U$. Since $p(\Im, \aleph) \subseteq\langle\Im, \aleph\rangle$, then $U \in\langle\Im, \aleph\rangle$. Hence, $(X, \Im, \aleph)$ is s-locally countable.

The following example shows that the converse of Theorem 4.3 is not true, in general.

Example 9. Let $X=\mathbb{R}$ and let $\Im$ and $\aleph$ be the topologies on $\mathbb{R}$ having respectively the families $\{[a, b): a, b \in \mathbb{R}$ and $a<b\}$ and $\{(a, b]: a, b \in \mathbb{R}$ and $a<b\}$ as bases. Then

(1) $(X, \Im, \aleph)$ is s-locally countable: let $x \in X$, then $x \in[x, x+1) \in \Im, x \in(x-1, x] \in \aleph$ and so $x \in[x, x+1) \cap(x-1, x]=\{x\} \in\langle\Im, \aleph\rangle$. Since $\{x\}$ is countable, we are done.

(2) $(X, \Im, \aleph)$ is not $p$-locally countable: by Theorem 4.2 , we only need to see that $(X, \Im, \aleph)$ is not u-locally countable. If $(X, \Im, \aleph)$ is u-locally countable, then there is $W \in u(\Im, \aleph)=\Im \cup \aleph$ such that $W$ is a countable set and $1 \in W$. Then, there is $[a, b)$ such that $1 \in[a, b) \subseteq W$ or there is $(a, b]$ such that $1 \in(a, b] \subseteq W$. Thus one of the sets $[a, b)$ and $(a, b]$ is countable, which is impossible.

Remark 5. If at least one of the TSs $(X, \Im)$ and $(X, \aleph)$ is locally countable, then the BTS $(X, \Im, \aleph)$ is u-locally countable.

Proof. Obvious.

The following example shows that the implication in Remark 5 is not reversible, in general.

Example 10. Let $X=\mathbb{R}, \mathcal{A}=\{\{x\}: x \in(-\infty, 0]\} \cup\{U \subseteq \mathbb{R}:(0, \infty) \subseteq U\}$ and $\mathcal{B}=\{\{x\}: x \in(0, \infty)\} \cup\{U \subseteq \mathbb{R}:(-\infty, 0] \subseteq U\}$. Let $\Im$ and $\aleph$ be the topologies on $\mathbb{R}$ having the families $\mathcal{A}$ and $\mathcal{B}$ as bases, respectively. Then, we have the following: 
(1) $(X, \Im, \aleph)$ is $u$-locally countable: let $x \in X$, then either $x \in(-\infty, 0]$ or $x \in(0, \infty)$. If $x \in(-\infty, 0]$, then we have $x \in\{x\} \in \mathcal{A} \subseteq \Im \subseteq u(\Im, \aleph)$ with $\{x\}$ is a countable set. If $x \in(0, \infty)$, then we have $x \in\{x\} \in \mathcal{B} \subseteq \aleph \subseteq u(\Im, \aleph)$ with $\{x\}$ is a countable set. It follows that $(X, \Im, \aleph)$ is u-locally countable.

(2) $(X, \Im)$ is not locally countable: if $(X, \Im)$ is locally countable, then there is $W \in \Im$ such that $W$ is a countable set and $1 \in W$. But by definition of $\Im$ we must have $(0, \infty) \subseteq W$ and so $W$ is uncountable. It follows that $(X, \Im)$ is not locally countable.

(3) $(X, \aleph)$ is not locally countable: if $(X, \aleph)$ is locally countable, then there is $W \in \aleph$ such that $W$ is a countable set and $0 \in W$. However, by the definition of $\aleph$, we must have $(-\infty, 0] \subseteq W$ and so $W$ is uncountable. It follows that $(X, \aleph)$ is not locally countable.

As defined in [2], a STS $(X, \tau, A)$ is soft locally countable if for any $a_{x} \in S P(X, A)$ there exists $H \in C S S(X, A) \cap \tau$ such that $a_{x} \widetilde{\in} H$.

Definition 13. A SBTS $(X, \tau, \sigma, A)$ is called the following:

(1) Soft u-locally countable if for each $a_{x} \in S P(X, A)$ there exists $H \in \operatorname{CSS}(X, A) \cap u(\tau, \sigma)$ such that $a_{x} \widetilde{\in} H$.

(2) Soft $p$-locally countable if for each $a_{x} \in S P(X, A)$, there exists $H \in \operatorname{CSS}(X, A) \cap p(\tau, \sigma)$ such that $a_{x} \widetilde{\in} H$.

(3) Soft s-locally countable if the STS $(X,\langle\tau, \sigma\rangle, A)$ is soft locally countable.

Theorem 15. A SBTS $(X, \tau, \sigma, A)$ is soft u-locally countable if and only if it is soft p-locally countable.

Proof. Necessity: Suppose that $(X, \tau, \sigma, A)$ is soft $u$-locally countable, and let $a_{x} \in S P(X, A)$. Then, there is $H \in \operatorname{CSS}(X, A) \cap u(\tau, \sigma)$ such that $a_{x} \widetilde{\in} H$. Since $u(\tau, \sigma) \subseteq p(\tau, \sigma)$, then $H \in p(\tau, \sigma)$. Hence, $(X, \tau, \sigma, A)$ is soft $p$-locally countable.

Sufficiency: Suppose that $(X, \tau, \sigma, A)$ is soft $p$-locally countable, and let $a_{x} \in \operatorname{SP}(X, A)$. Then there is $H \in C S S(X, A) \cap p(\tau, \sigma)$ such that $a_{x} \widetilde{\in} H$. Since $H \in p(\tau, \sigma)$, then there exist $M \in \tau$ and $N \in \sigma$ such that $H=M \widetilde{\cup} N$. Therefore, we have $M \in C S S(X, A) \cap u(\tau, \sigma)$ such that $a_{x} \widetilde{\in} M$ or we have $N \in \operatorname{CSS}(X, A) \cap u(\tau, \sigma)$ such that $a_{x} \widetilde{\in} N$. Hence, $(X, \tau, \sigma, A)$ is soft $u$-locally countable.

Theorem 16. Every soft p-locally countable SBTS is soft s-locally countable.

Proof. Let $(X, \tau, \sigma, A)$ be soft $p$-locally countable, and let $a_{x} \in S P(X, A)$. Then, there is $H \in \operatorname{CSS}(X, A) \cap p(\tau, \sigma)$ such that $a_{x} \widetilde{\in} H$. Since $p(\tau, \sigma) \subseteq\langle\tau, \sigma\rangle$, then $H \in\langle\tau$, $\sigma\rangle$. Hence, $(X, \tau, \sigma, A)$ is soft $s$-locally countable.

The following example shows that the implication in Theorem 16 is not reversible, in general.

Example 11. Let $X=\mathbb{R}$ and $A=\mathbb{N}$. Let $\Im$ and $\aleph$ be the topologies on $\mathbb{R}$ having the families $\{[a, b): a, b \in \mathbb{R}$ and $a<b\}$ and $\{(a, b]: a, b \in \mathbb{R}$ and $a<b\}$ as bases, respectively. Let $\tau=$ $\{F \in S S(X, A): F(a) \in \Im$ for all $a \in A\}$ and $\sigma=\{F \in S S(X, A): F(a) \in \aleph$ for all $a \in A\}$. Then

(1) $(X, \tau, \sigma, A)$ is soft s-locally countable: let $a_{x} \in S P(X, A)$. Let $H, K \in S S(X, A)$ defined by $H(a)=[x, x+1)$ and $K(a)=(x-1, x]$ for all $a \in A$, then $(H \widetilde{\cap} K)(a)=\{x\}$. Thus, we have $H \widetilde{\cap} K \in \operatorname{CSS}(X, A) \cap\langle\tau, \sigma\rangle$ with $a_{x} \widetilde{\in} H \widetilde{\cap} K$. It follows that $(X, \tau, \sigma, A)$ is soft s-locally countable.

(2) $(X, \tau, \sigma, A)$ is not soft p-locally countable: by Theorem 4.8 , only we need to see that $(X, \tau, \sigma, A)$ is not soft $u$-locally countable. If $(X, \tau, \sigma, A)$ is soft u-locally countable, then there is $H \in \operatorname{CSS}(X, A) \cap u(\tau, \sigma)$ such that $1_{2} \widetilde{\in} H$. Then, there is $[a, b)$ such that $2 \in[a, b) \subseteq H(1)$ or there is $(a, b]$ such that $2 \in(a, b] \subseteq H(1)$. Thus, one of the sets $[a, b)$ and $(a, b]$ is countable, which is impossible. 
Remark 6. If at least one of the STSs $(X, \tau, A)$ and $(X, \sigma, A)$ is soft locally countable, then the $\operatorname{SBTS}(X, \tau, \sigma, A)$ is soft u-locally countable.

Proof. Obvious.

The following example shows that the implication in Remark 6 is not reversible, in general.

Example 12. Let $X=\mathbb{R}$ and $A=\mathbb{Z}$. Let $\mathcal{A}=\{\{x\}: x \in(-\infty, 0]\} \cup\{U \subseteq \mathbb{R}:(0, \infty) \subseteq U\}$ and $\mathcal{B}=\{\{x\}: x \in(0, \infty)\} \cup\{U \subseteq \mathbb{R}:(-\infty, 0] \subseteq U\}$. Let $\Im$ and $\aleph$ be the topologies on $\mathbb{R}$ having the families $\mathcal{A}$ and $\mathcal{B}$ as bases, respectively. Let $\tau=\{F \in S S(X, A): F(a) \in \Im$ for all $a \in A\}$ and $\sigma=\{F \in S S(X, A): F(a) \in \aleph$ for all $a \in A\}$. Then, we have the following:

(1) $(X, \tau, \sigma, A)$ is soft $u$-locally countable: let $b_{x} \in S P(X, A)$. We have two cases on $x$ :

Case 1. $x \in(-\infty, 0]$. Let $H \in S S(X, A)$ defined by $H(a)=\{x\}$ for all $a \in A$. Then, we have $H \in \operatorname{CSS}(X, A)$ with $a_{x} \widetilde{\in} H$ and $H \in \tau \subseteq u(\tau, \sigma)$.

Case 2. $x \in(0, \infty)$. Let $H \in S S(X, A)$ defined by $H(a)=\{x\}$ for all $a \in A$. Then, we have $H \in \operatorname{CSS}(X, A)$ with $a_{x} \widetilde{\in} H$ and $H \in \sigma \subseteq u(\tau, \sigma)$.

It follows that $(X, \tau, \sigma, A)$ is soft $u$-locally countable.

(2) $(X, \tau, A)$ is not soft locally countable: if $(X, \tau, A)$ is soft locally countable, then there is $H \in \operatorname{CSS}(X, A) \cap \tau$ such that $1_{2} \widetilde{\in} H$. Then $2 \in H(1)$ and $H(1)$ is a countable set. However, by the definition of $\tau$, we must have $(0, \infty) \subseteq H(1)$ and hence $H(1)$ is uncountable. It follows that $(X, \tau, A)$ is not soft locally countable.

(3) $(X, \sigma, A)$ is not soft locally countable: if $(X, \sigma, A)$ is soft locally countable, then there is $H \in \operatorname{CSS}(X, A) \cap \sigma$ such that $1_{0} \widetilde{\in} H$. Then $0 \in H(1)$ and $H(1)$ is a countable set. However, by the definition of $\sigma$, we must have $(-\infty, 0] \subseteq H(1)$ and hence $H(1)$ is uncountable. It follows that $(X, \tau, A)$ is not soft locally countable.

Theorem 17. If $(X, \tau, A)$ is soft locally countable, then $\left(X, \tau_{a}\right)$ is locally countable for all $a \in A$.

Proof. Suppose that $(X, \tau, A)$ is soft locally countable and let $a \in A$. Let $x \in X$, then $a_{x} \in S P(X, A)$. By the soft local countability of $(X, \tau, A)$, there exists $H \in C S S(X, A) \cap \tau$ such that $a_{x} \widetilde{\in} H$. Then, we have $x \in H(a) \in \tau_{a}$ and $H(a)$ is a countable set. This shows that $\left(X, \tau_{a}\right)$ is locally countable.

Corollary 3. Let $X$ be an initial universe and let $A$ be a set of parameters. Let $\left\{\Im_{a}: a \in A\right\}$ be an indexed family of topologies on $X$. If $\left(X, \oplus \Im_{a}, A\right)$ is soft locally countable, then $\left(X, \Im_{a}\right)$ is locally countable for all $a \in A$.

Proof. Suppose that $\left(X, \underset{a \in A}{\oplus} \Im_{a}, A\right)$ is soft locally countable and let $a \in A$. Then by Theorem 17, $\left(X,\left(\bigoplus_{a \in A} \Im_{a}\right)_{a}\right)$. However, by Theorem 3.7 of [1], $\left(\bigoplus_{a \in A} \Im_{a}\right)_{a}=\Im_{a}$. This ends the proof.

Theorem 18. If $A$ is countable and $\left(X, \tau_{a}\right)$ is locally countable for all $a \in A$, then $(X, \tau, A)$ is soft locally countable.

Proof. Suppose that $\left(X, \tau_{a}\right)$ is locally countable for all $a \in A$ such that $A$ is countable. Let $b_{x} \in S P(X, A)$. For every $a \in A$, by the local countability of $\left(X, \tau_{a}\right)$, there exists $H_{a} \in \tau$ such that $x \in H_{a}(a) \in \tau_{a}$ and $H_{a}(a)$ is countable. Put $H=\widetilde{\cup}{ }_{a \in A} H_{a}$; then, $H \in C S S(X, A) \cap \tau$ and $b_{x} \widetilde{\in} H$. Therefore, $(X, \tau, A)$ is soft locally countable.

Corollary 4. Let $X$ be an initial universe and let $A$ be a countable set of parameters. Let $\left\{\Im_{a}: a \in A\right\}$ be an indexed family of topologies on $X$. If $\left(X, \Im_{a}\right)$ is locally countable for all $a \in A$, then $\left(X, \bigoplus_{a \in A} \Im_{a}, A\right)$ is soft locally countable. 
Proof. Suppose that $\left(X, \Im_{a}\right)$ is locally countable for all $a \in A$. By Theorem 3.7 of [1], $\left(\underset{a \in A}{\oplus} \Im_{a}\right)_{a}=\Im_{a}$ and so by Theorem 18 , we must have $\left(X, \underset{a \in A}{\oplus} \Im_{a}, A\right)$ is soft locally countable.

Theorem 19. If $(X, \tau, \sigma, A)$ is soft u-locally countable, then $\left(X, \tau_{a}, \sigma_{a}\right)$ is u-locally countable for all $a \in A$.

Proof. Suppose that $(X, \tau, \sigma, A)$ is soft $u$-locally countable and let $a \in A$. Let $x \in X$, then $a_{x} \in \operatorname{SP}(X, A)$. By soft $u$-local countability of $(X, \tau, \sigma, A)$, there exists $H \in \operatorname{CSS}(X, A) \cap$ $u(\tau, \sigma)$ such that $a_{x} \widetilde{\in} H$. Then we have $H(a)$ is a countable set, $x \in H(a)$, and by Theorem 6 (a) we have $H(a) \in u\left(\tau_{a}, \sigma_{a}\right)$. Therefore, $\left(X, \tau_{a}, \sigma_{a}\right)$ is $u$-locally countable.

Corollary 5. Let $X$ be an initial universe and $A$ be a set of parameters. Let $\left\{\Im_{a}: a \in A\right\}$ and $\left\{\aleph_{a}: a \in A\right\}$ be indexed families of topologies on X. If $\left(X, \underset{a \in A}{\oplus} \Im_{a}, \underset{a \in A}{\oplus} \aleph_{a}, A\right)$ is soft u-locally countable; then, $\left(X, \Im_{a}, \aleph_{a}\right)$ is u-locally countable for all $a \in A$.

Proof. Suppose that $\left(X, \underset{a \in A}{\oplus} \Im_{a}, \underset{a \in A}{\oplus} \aleph_{a}, A\right)$ is soft $u$-locally countable and let $a \in A$. Then, by Theorem 4.17, $\left(X,\left(\underset{a \in A}{\oplus} \Im_{a}\right)_{a},\left(\underset{a \in A}{\oplus} \aleph_{a}\right)_{a}\right)$ is $u$-locally countable. However, by Theorem 3.7 of [1], $\left(\underset{a \in A}{\oplus} \Im_{a}\right)_{a}=\Im_{a}$ and $\left(\underset{a \in A}{\oplus} \aleph_{a}\right)_{a}=\aleph_{a}$. This ends the proof.

Theorem 20. If $A$ is countable and $\left(X, \tau_{a}, \sigma_{a}\right)$ is p-locally countable for all $a \in A$; then, $(X, \tau, \sigma, A)$ is soft $p$-locally countable.

Proof. Suppose that $\left(X, \tau_{a}, \sigma_{a}\right)$ is $p$-locally countable for all $a \in A$ and let $b_{x} \in \operatorname{SP}(X, A)$. For every $a \in A$, by $p$-local countability of $\left(X, \tau_{a}, \sigma_{a}\right)$, there exists $H_{a} \in p(\tau, \sigma)$ such that $H_{a}(a) \in p\left(\tau_{a}, \sigma_{a}\right)$ and $H_{a}(a)$ is countable. Put $H=\widetilde{\cup}\left\{H_{a}: a \in A\right\}$; then $H \in \operatorname{CSS}(X, A) \cap$ $p(\tau, \sigma)$ and $b_{x} \tilde{\in} H$. Therefore, $(X, \tau, \sigma, A)$ is soft $p$-locally countable.

Corollary 6. If $A$ is countable and $\left(X, \tau_{a}, \sigma_{a}\right)$ is u-locally countable for all $a \in A$, then $(X, \tau, \sigma, A)$ is soft u-locally countable.

Proof. This follows from Theorems 13, 15, and 20.

Corollary 7. Let $X$ be an initial universe and let $A$ be a countable set of parameters. Let $\left\{\Im_{a}: a \in A\right\}$ and $\left\{\aleph_{a}: a \in A\right\}$ be indexed families of topologies on X. If $\left(X, \Im_{a}, \aleph_{a}\right)$ is u-locally countable for all $a \in A$, then $\left(X, \underset{a \in A}{\oplus} \Im_{a}, \underset{a \in A}{\oplus} \aleph_{a}, A\right)$ is soft u-locally countable.

Proof. Suppose that $\left(X, \Im_{a}, \aleph_{a}\right)$ is $u$-locally countable for all $a \in A$. By Theorem 3.7 of [1], $\left(\underset{a \in A}{\oplus} \Im_{a}\right)_{a}=\Im_{a}$ and $\left(\underset{a \in A}{\oplus} \aleph_{a}\right)_{a}=\aleph_{a}$. So by Corollary 6, we must have $\left(X, \underset{a \in A}{\oplus} \Im_{a}, \underset{a \in A}{\oplus} \aleph_{a}, A\right)$ is soft $u$-locally countable.

Theorem 21. A SBTS $(X, \tau, \sigma, A)$ is soft $p$-locally countable if and only if $\operatorname{SP}(X, A) \subseteq p \omega(\tau, \sigma)$.

Proof. Necessity: Suppose that $(X, \tau, \sigma, A)$ is soft $p$-locally countable. Let $a_{x} \in \operatorname{SP}(X, A)$. Then by soft $p$-local countability of $(X, \tau, \sigma, A)$, there exists $H \in \operatorname{CSS}(X, A) \cap p(\tau, \sigma)$ such that $a_{x} \widetilde{\in} H$. Since $H \in \operatorname{CSS}(X, A)$, then $H-a_{x} \in \operatorname{CSS}(X, A)$. Thus, we have $a_{x}=$ $\left(H-\left(H-a_{x}\right)\right) \in p \omega(\tau, \sigma)$.

Sufficiency: Suppose that $S P(X, A) \subseteq p \omega(\tau, \sigma)$. Let $a_{x} \in S P(X, A)$, then $a_{x} \in p \omega(\tau, \sigma)$. So, there are $M \in \tau_{\omega}$ and $N \in \sigma_{\omega}$ such that $a_{x}=M \widetilde{\cup} N$. So, $a_{x}=M$ or $a_{x}=N$. Hence, there 
is $G \in \tau \cup \sigma$ and $H \in \operatorname{CSS}(X, A)$ such that $a_{x} \widetilde{\in} G-H \widetilde{\widetilde{C}} a_{x}$. Hence, $G \in \operatorname{CSS}(X, A) \cap p(\tau, \sigma)$ with $a_{x} \tilde{\in} G$. Therefore, $(X, \tau, \sigma, A)$ is soft $p$-locally countable.

Corollary 8. A SBTS $(X, \tau, \sigma, A)$ is soft $p$-locally countable if and only if $(X, p \omega(\tau, \sigma), A)$ is a discrete STS.

Theorem 22. If $(X, \tau, \sigma, A)$ is soft s-locally countable, then $\left(X, \tau_{a}, \sigma_{a}\right)$ is s-locally countable for all $a \in A$.

Proof. Suppose that $(X, \tau, \sigma, A)$ is soft s-locally countable and let $a \in A$. Let $x \in X$, then $a_{x} \in \operatorname{SP}(X, A)$. By soft $s$-local countability of $(X, \tau, \sigma, A)$, there exists $H \in \operatorname{CSS}(X, A) \cap$ $\langle\tau, \sigma\rangle$ such that $a_{x} \widetilde{\in} H$. Then, we have $H(a)$ is a countable set, $x \in H(a)$, and by Theorem 6 (c) we have $H(a) \in\left\langle\tau_{a}, \sigma_{a}\right\rangle$. Therefore, $\left(X, \tau_{a}, \sigma_{a}\right)$ is s-locally countable.

Theorem 23. If $A$ is countable and $\left(X, \tau_{a}, \sigma_{a}\right)$ is is s-locally countable for all $a \in A$, then $(X, \tau, \sigma, A)$ is soft s-locally countable.

Proof. Suppose $\left(X, \tau_{a}, \sigma_{a}\right)$ is s-locally countable for all $a \in A$ such that $A$ is countable. Let $b_{x} \in S P(X, A)$. For every $a \in A$, by s-local countability of $\left(X, \tau_{a}, \sigma_{a}\right)$, there exists $H_{a} \in\langle\tau, \sigma\rangle$ such that $H_{a}(a) \in\left\langle\tau_{a}, \sigma_{a}\right\rangle$ and $H_{a}(a)$ is countable. Put $H=\underset{a \in A}{\widetilde{U}} H_{a}$, then $H \in \operatorname{CSS}(X, A) \cap\langle\tau, \sigma\rangle$ and $b_{x} \tilde{\in} H$. Therefore, $(X, \tau, \sigma, A)$ is soft s-locally countable.

Theorem 24. A SBTS $(X, \tau, \sigma, A)$ is soft s-locally countable if and only if $\left(X,\left\langle\tau_{\omega}, \sigma_{\omega}\right\rangle, A\right)$ is a discrete STS.

Proof. $(X, \tau, \sigma, A)$ if and only if $(X,\langle\tau, \sigma\rangle, A)$ is soft locally countable, and by Corollary 5 of [2] just this holds if and only if $\left(X,\langle\tau, \sigma\rangle_{\omega}, A\right)$ is a discrete STS. So, by Theorem 7 (f), we obtain the result.

\section{Conclusions}

As modifications of soft $p$-open sets, five new classes of soft sets in SBTSs were investigated, and via them, several types soft Lindelöfeness and soft local countability in SBTSs were defined and studied. Correspondence between SBTSs and their generated BTSs were introduced. In future studies, the following topics could be considered: (1) defining soft $u$-continuity, soft $s$-continuity, $u$ - $\omega$-continuity, soft $p$ - $\omega$-continuity, and soft $s-\omega$-continuity; and (2) defining separation axioms via these new five classes of soft sets.

Funding: This research received no external funding.

Institutional Review Board Statement: Not applicable.

Informed Consent Statement: Not applicable.

Data Availability Statement: Not applicable.

Conflicts of Interest: The author declare no conflict of interest.

\section{References}

1. Al Ghour, S.; Bin-Saadon, A. On some generated soft topological spaces and soft homogeneity. Heliyon 2019, 5, e02061. [CrossRef]

2. Al Ghour, S.; Hamed, W. On two classes of soft sets in soft topological spaces. Symmetry 2020, 12, 265. [CrossRef]

3. Molodtsov, D. Soft set theory-First results. Comput. Math. Appl. 1999, 37, 19-31. [CrossRef]

4. Shabir, M.; Naz, M. On soft topological spaces. Comput. Math. Appl. 2011, 61, 1786-1799. [CrossRef]

5. Al Ghour, S. Soft $\omega^{*}$-paracompactness in soft topological spaces. Int. J. Fuzzy Log. Intell. Syst. 2021, 21, 57-65. [CrossRef]

6. Mousarezaei, R.; Davvaz, B. On Soft Topological Polygroups and Their Examples. Int. J. Fuzzy Log. Intell. Syst. 2021, $21,29-37$. [CrossRef]

7. Oztunc, S.; Aslan, S.; Dutta, H. Categorical structures of soft groups. Soft Comput. 2021, 25, 3059-3064. [CrossRef]

8. Demİr, I. Some soft topological properties and fixed soft element results in soft complex valued metric spaces. Turk. Math. 2021, 45, 971-987. [CrossRef] 
9. Al-Shami, T.M.; Abo-Tabl, E.S.A. Soft $\alpha$-separation axioms and $\alpha$-fixed soft points. AIMS Math. 2021, 6, 5675-5694. [CrossRef]

10. Al-Shami, T.M. On soft separation axioms and their applications on decision-making problem. Math. Probl. Eng. 2021, 2021, 8876978. [CrossRef]

11. Al-shami, T.M. Bipolar soft sets: Relations between them and ordinary points and their applications. Complexity 2021, 2021, 6621854. [CrossRef]

12. Al-shami, T.M.; Alshammari, I.; Asaad, B.A. Soft maps via soft somewhere dense sets. Filomat 2020, 34, 3429-3440. [CrossRef]

13. Oguz, G. Soft topological transformation groups. Mathematics 2020, 8, 1545. [CrossRef]

14. Min, W.K. On Soft $\omega$-Structures Defined by Soft Sets. Int. J. Fuzzy Logic Intell. Syst. 2020, 20, 119-123. [CrossRef]

15. V. Cetkin, V.; Guner, E.; Aygün, H. On 2S-metric spaces. Soft Comput. 2020, 24, 12731-12742. [CrossRef]

16. El-Shafei, M.E.; Al-shami, T.M. Applications of partial belong and total non-belong relations on soft separation axioms and decision-making problem. Comput. Appl. Math. 2020, 39, 138. [CrossRef]

17. Alcantud, J.C.R. Soft open bases and a novel construction of soft topologies from bases for topologies. Mathematics $2020,8,672$. [CrossRef]

18. Bahredar, A.A.; Kouhestani, N. On $\varepsilon$-soft topological semigroups. Soft Comput. 2020, 24, 7035-7046. [CrossRef]

19. Al-shami, T.M.; El-Shafei, M.E. Partial belong relation on soft separation axioms and decision-making problem, two birds with one stone. Soft Comput. 2020, 24, 5377-5387. [CrossRef]

20. Al-shami, T.M.; Kocinac, L.; Asaad, B.A. Sum of soft topological spaces. Mathematics 2020, 8, 990. [CrossRef]

21. Al-shami, T.M. Soft somewhere dense sets on soft topological spaces. Commun. Korean Math. Soc. 2018, 33, 1341-1356.

22. Al-Saadi, H.S.; Min, W.K. On soft generalized closed sets in a soft topological space with a soft weak structure. Int. Fuzzy Log. Intell. Syst. 2017, 17, 323-328. [CrossRef]

23. Hdeib, H. $\omega$-Closed mappings. Rev. Colomb. Math. 1982, 16, 65-78.

24. Al Ghour, S.; Al-Zoubi, S. A new class between theta open sets and theta omega open sets. Heliyon 2021, 7, e05996. [CrossRef]

25. Al Ghour, S.; Irshidat, B. On $\theta_{\omega}$-continuity. Heliyon 2020, 6, e03349. [CrossRef]

26. Latif, R.M. Theta- $\omega$-Mappings in topological spaces. WSEAS Trans. Math. 2020, 19, 186-207. [CrossRef]

27. Al Ghour, S.; El-Issa, S. $\theta_{\omega}$-Connectedness and $\omega$ - $R_{1}$ properties. Proyecciones 2019, 38, 921-942. [CrossRef]

28. Carpintero, C.; Rajesh, N.; Rosas, E. On real valued $\omega$-continuous functions. Acta Univ. Sapientiae, Math.2018, 10, 242-248. [CrossRef]

29. Al Ghour, S. Theorems on Strong Paracompactness of Product Spaces. Math. Notes 2018, 103, 54-58. [CrossRef]

30. Al Ghour, S.; Issa, S. On $u$ - $\omega$-open and $p$ - $\omega$-open sets in bitopological spaces. Mo. J. Math. 2012, 24, 37-53. [CrossRef]

31. Granados, C. On soft $b-\omega$-open sets. J. Indones. Math. Soc. 2021, 37, 123-129. [CrossRef]

32. Ittanagi, B.M. Soft bitopological spaces. Int. Comput. Appl. 2014, 107, 1-4.

33. Sayed, A.F. On $(1,2)^{*}$-fuzzy soft $b$-continuity in fuzzy soft bitopological spaces. Soft Comput. 2021, in press. [CrossRef]

34. Mehmood, A.; Al-Shomrani, M.M.; Zaighum, M.A.; Abdullah, S. Characterization of soft S-open sets in Bi-soft topological structure concerning crisp points. Mathematics 2020, 8, 2100. [CrossRef]

35. Sayed, A.F. On fuzzy soft B-open sets in fuzzy soft bitopological space. J. Math. Comput. Sci.2020, 21, 31-44. [CrossRef]

36. Kandil, A.; Tantawy, O.A.E.; El-Sheikh, S.A.; Hazza, S.A. Pairwise open (closed) soft sets in soft bitopological spaces. Ann. Fuzzy Math. Inform. 2016, 11, 1-20.

37. Kilicman, A.; Salleh, Z. A note on pairwise continuous mappings and bitopological spaces. Eur. J. Pure Appl. Math. 2009, 2, 325-337.

38. Datta, M. Projective bitopological spaces. J. Aust. Math. Soc. 1972, 13, 327-334. [CrossRef]

39. Maji, P.K.; Biswas, R.; Roy, A.R. Soft set theory. Comput. Math. Appl. 2003, 45, 555-562. [CrossRef]

40. Zorlutuna, I.; Akdag, M.; Min, W.K.; Atmaca, S. Remarks on soft topological spaces. Ann. Fuzzy Math. Inform. $2012,3,171-185$.

41. Das, S.; Samanta, S.K. Soft metric. Ann. Fuzzy Math. Inform. 2013, 6, 77-94. 